\title{
Longitudinal Stability Evaluation of Biomarkers and Their Correlation in Cerebrospinal Fluid and Plasma from Patients with Alzheimer's Disease
}

\author{
Kina Höglund ${ }^{\mathrm{a}, *}$, Anna Bogstedt ${ }^{\mathrm{a}}$, Susanne Fabre ${ }^{\mathrm{a}}$, Ali Aziz ${ }^{\mathrm{a}}$, Peter Annas ${ }^{\mathrm{a}}$, Hans Basun ${ }^{\mathrm{b}}$, \\ Lennart Minthon $^{\mathrm{c}}$, Lars Lannfelt ${ }^{\mathrm{b}}$, Kaj Blennow ${ }^{\mathrm{d}}$ and Niels Andreasen ${ }^{\mathrm{e}}$ \\ ${ }^{a}$ AstraZeneca Translational Sciences Centre, Science for Life Laboratory, Solna, Sweden \\ ${ }^{\mathrm{b}}$ Department of Public Health and Caring Sciences, Uppsala University, Uppsala, Sweden \\ ${ }^{\mathrm{c} C l i n i c a l}$ Memory Research Unit, Department of Clinical Sciences Malmö, Lund University, Sweden \\ ${ }^{\mathrm{d}}$ Clinical Neurochemistry Laboratory, The Sahlgrenska Academy at Gothenburg University, Mölndal, Sweden \\ ${ }^{\mathrm{e}}$ Karolinska Institutet Alzheimer Disease Research Center, Department NVS för KI och Geriatric Clinic, \\ Karolinska University Hospital, Stockholm, Sweden
}

Handling Associate Editor: Panos Alexopoulos

Accepted 29 June 2012

\begin{abstract}
There is an increasing demand for biomarkers in clinical treatment trials to demonstrate target engagement and to support disease modification claims. To be able to detect treatment related effects, a prerequisite is that the levels of the biomarker are stable over time or that the change over time is known. In the present study, the stability of $\alpha$ - and $\beta$-cleaved soluble amyloid- $\beta$ protein precursor ( $\mathrm{sA} \beta \mathrm{PP} \alpha$ and $\mathrm{sA} \beta \mathrm{PP} \beta), \mathrm{A} \beta_{1-40}$ together with the phosphorylated form of neurofilament heavy/medium ( $\mathrm{pNfH} / \mathrm{M})$ in cerebrospinal fluid (CSF) was analyzed in a cohort of 51 patients with Alzheimer's disease. In addition, the stability of $A \beta_{1-40}, A \beta_{1-42}$, and $\mathrm{A} A \mathrm{PP} \beta$ in plasma was explored. Plasma and CSF was sampled at baseline and after 6-months follow up, and all patients were on stable treatment with acetylcholinesterase inhibitors. During this 6-month longitudinal follow-up, we saw a small, but consistent and statistically significant increase in CSF levels of sA $\beta$ PP $\beta$ (103\% of baseline levels) and a statistically significant decrease in the CSF levels of $\mathrm{pNfH} / \mathrm{M}$ ( $91 \%$ of baseline levels). The mean level of the CSF biomarkers were very stable between baseline and endpoint, with within-patients coefficients of variation (CVs) of $5.84-17.3 \%$, while the variability was larger for the plasma biomarkers, with CVs of $14.1-42.3 \%$. This stability suggests that these biomarkers may have the potential to detect and monitor biochemical changes induced by disease-modifying drugs.
\end{abstract}

Keywords: Alzheimer's disease, biomarkers, cerebrospinal fluid, longitudinal, plasma, stability

\section{INTRODUCTION}

Alzheimer's disease (AD) is the most common form of dementia, affecting more than $50 \%$ of people

\footnotetext{
*Correspondence to: Kina Höglund, Translational Science Centre, Karolinska Institute, Tomtebodavägen 23B, Floor 4, S-171 65 Solna, Sweden. Tel.: +46 8553 23283; Mob.: +46 763074 975; E-mail: kina.hoglund@astrazeneca.com.
}

aged 80. With increasing lifespan, AD is becoming a major health problem for society. Clinical diagnosis can be made with approximately $80-90 \%$ accuracy, while definite diagnosis only can be made through postmortem neuropathological examination. The two major brain pathological hallmarks of $\mathrm{AD}$ are senile plaques and neurofibrillary tangles. The plaques are composed of amyloid peptide while tau, a microtubule 
binding protein, is the building block of tangles. These pathological findings have paved the way for many treatment hypotheses which have initiated drug discovery projects aiming at identifying a disease modifying drug. Being able to claim disease modification improved cognition or halted cognitive decline needs to be supported by biomarkers reflecting effects on disease pathology.

Three proteins linked to pathology are established biomarkers for diagnosing $\mathrm{AD}$; the total levels of tau (t-tau), the phosphorylated variant (p-tau), and the amyloid- $\beta(A \beta)_{1-42}$ peptide. The cerebrospinal fluid (CSF) levels of t-tau and $\mathrm{p}$-tau are found to be increased 200-300\% in patients with AD compared to controls $[1,2]$. These elevations are believed to reflect neuronal degeneration [3]. The levels of $A \beta_{1-42}$ are found to be decreased in patients with $\mathrm{AD}$ [4], and there is evidence today that this decrease reflects the accumulation of $A \beta$ into the extracellular senile plaques [5, $6]$. The $A \beta$ peptide is produced during the processing of the amyloid- $\beta$ protein precursor (A $\beta P P)$ and two major pathways have been described. The major pathway includes proteolytic processing of A $\beta P P$ by $\alpha$ - and $\gamma$-secretase, precluding the formation of $\mathrm{A} \beta$ peptide. The second pathway includes the proteolytical cleavage by $\beta$ - and $\gamma$-secretase, producing the $A \beta$ peptide. The major $A \beta$ species produced are $A \beta_{1-40}$, followed by $A \beta_{1-38}$ and $A \beta_{1-42}$. However, truncations at both $\mathrm{N}$ - and $\mathrm{C}$-terminal end of $\mathrm{A} \beta$ occurs and many different $\mathrm{A} \beta$ species are present both in blood, brain, and CSF [7-9].

Biomarkers to monitor treatment effects, either related to target (mechanistic markers) or to disease pathology (proof of principle markers) are needed to evaluate drug response. The stability of that biomarker, whether it is progressing with disease state or not, is of importance in order to be able to predict outcome of a successful treatment. CSF fills the ventricles and surrounds the external surface of the brain and is directly connected to the extracellular (interstitial) fluid [10]. The extracellular fluid surrounds neurons and glia and due to this close contact between brain and CSF, pathological changes in the brain are believed to be reflected in the CSF, as measured by proteins, lipids, or neuropeptides. Proteins found in the CSF are predominantly produced in the central nervous system (CNS) and are not influenced by blood concentrations or blood-brain barrier permeability; hence very seldom are CSF levels of these brain specific proteins related to blood levels. The search for pathologically related biomarkers in the plasma is therefore challenging. However, read-outs in plasma to demonstrate peripheral target engagement in early clinical trials are of value.

In the present study we have examined the stability of $\alpha$ - and $\beta$-cleaved $\operatorname{sA} \beta P P(\operatorname{sA} \beta P P \alpha$ and sA $\beta P P \beta$, respectively) and $A \beta_{1-40}$ in CSF together with $\mathrm{SA} \beta \mathrm{PP} \beta, A \beta_{1-40}$, and $A \beta_{1-42}$ in plasma over a 6 month period in patients with AD. Levels of sA $\beta P P \beta$, $A \beta_{1-42}$, and $A \beta_{1-40}$ are possible mechanistic markers when inhibiting BACE, the enzyme responsible for producing $A \beta$. Levels of $\mathrm{sA} \beta P P \beta$ in plasma will provide information on peripheral target engagement while levels of sA $\beta P P \beta$ in CSF provide information on central target engagement, which is a key read-out to identify a successful treatment in neurodegenerative disorders. The present study also includes evaluation of the stability of CSF levels of the phosphorylated form of neurofilament heavy and medium chain $(\mathrm{NfH} / \mathrm{M})$. Neurofilaments (Nf) are the major axonal cytoskeletal protein and found exclusively in neurons [11], hence CSF levels of $\mathrm{Nf}$ are potential markers of axonal degeneration, linked to disease pathology. The Nf protein is a heteropolymer composed of three subunits, $\mathrm{Nf}$ light (NfL), medium (NfM), and heavy (NfH) [12]. The NfL is the most abundant form (ratio: $4: 2: 1$, $\mathrm{NfL}: \mathrm{NfM}: \mathrm{NfH}$ ) containing only four sites for phosphorylation. $\mathrm{NfH}$ contains more than 100 potential phosphorylation sites and might therefore be extensively phosphorylated. The phosphorylation degree of the protein has been shown to correlate to decreased susceptibility to proteases [13]. In accordance with this, NfL levels in CSF tends to be very unstable which reduces its reliability as a biomarker to monitor treatment effects. Earlier studies have shown that Nfs might work as a diagnostic marker for AD where both NfL and NfH have been studied [14-16].

In the present study we provide a longitudinal follow-up of selected biomarkers in CSF and plasma which in general demonstrate very stable levels over time. However, a small but statistically significant change of CSF levels of $\mathrm{pNfH} / \mathrm{M}$ and $\mathrm{sA} \beta \mathrm{PP} \beta$ was observed. The $\mathrm{pNfH} / \mathrm{M}$ decreased by $9 \%$ comparing endpoint with baseline while CSF levels of sA $\beta P P \beta$ increased by $3 \%$. There was no correlation to APOE4 status or gender and we found no correlation between $\mathrm{pNfH} / \mathrm{M}$ or sA $\beta \mathrm{PP} \beta$ changes and the cognitive status at baseline. To our knowledge this is the first time longitudinal data are reported on CSF levels of $\mathrm{pNfH} / \mathrm{M}$, $\mathrm{SA} \beta P P \alpha$, and $\mathrm{SA} \beta \mathrm{PP} \beta$ in patients with $\mathrm{AD}$. This is also the first time data on $\mathrm{SA} \beta P P \beta$ in human plasma are published. As for plasma levels of $A \beta_{1-40}$ and $A \beta_{1-42}$, there is a large intra-individual variance of $\mathrm{sA} \beta \mathrm{PP} \beta$, but plasma levels are very stable over time. 


\section{MATERIALS AND METHODS}

The study was a 6-month three-center open study on patients with $\mathrm{AD}$ (26 men and 25 women) on continuous treatment with acetylcholine esterase (AChE) inhibitors. All patients underwent a thorough clinical investigation which included a medical history, physical, and neurological and psychiatric examinations. $\mathrm{AD}$ was diagnosed following the guidelines in the DSM-IV [17] and NINCDS-ADRDA criteria [18]. All patients with $\mathrm{AD}$ had an insidious onset and even progression of cognitive symptoms, which could not be explained by other systemic or brain disorders than AD. No patient had prominent frontal lobe symptoms, or history, clinical or brain imaging signs of cerebrovascular disease, except for mild white-matter lesions. No patient with AD had a family history of dementia suggestive of autosomal dominant AD.

At the first visit for screening and enrolment, eligibility criteria were checked and a mini-mental state examination (MMSE) [19] was performed. The inclusion criteria were AD of mild to moderate severity (MMSE score $>15$ ) and continuous treatment with AChE inhibitors at stable doses for at least 3 months prior to the study. Exclusion criteria were other concomitant diseases likely to interfere with study objectives as judged by the investigator, treatment with lithium, warfarin, ormemantine, and also medication with CNS active substances, such as anti-depressants or neuroleptics, less than 3 months prior to the study. Patients were withdrawn from the study if they were not able to complete the cognitive tests, or if there was a failure to obtain two CSF samples without major blood contamination. Subjects were also free to discontinue their participation in the study at any time. Adverse events were recorded during the treatment period. At the second visit, within 4 weeks after enrollment, the cognitive tests MMSE and Alzheimer's disease Assessment Scale, Cognitive subscale (ADASCog) [20] were performed, and a CSF sample was obtained. At the third visit, 6 months later, cognitive tests were repeated and a second CSF sample was obtained.

In total, $65 \mathrm{AD}$ cases were screened for the study. Six cases discontinued after visit 2, due to adverse events or that they were not willing to continue, leaving 59 cases that completed the study. Six cases were excluded since their dosing of AChE inhibitors had been changed during the study and another patient was excluded due to loss of the follow up sample and a final patient excluded due to no sample, leaving 51 cases, 26 men and 25 women. The mean age was 75.7
Table 1

Demographic data

\begin{tabular}{lcc}
\hline & Baseline & Endpoint \\
\hline Treatment (Riv/Gal/Don) & $7 / 22 / 22$ & $7 / 22 / 22$ \\
MMSE & $24.2(16-30)$ & $22.9(15-30)$ \\
ADAS-Cog & $14.3(1.3-31.7)$ & $14.2(1.0-30.3)$ \\
\hline
\end{tabular}

Data for the Mini-Mental Status Examination (MMSE) and the Alzheimer's Disease Assessment Scale, cognitive subscale (ADAS$\mathrm{Cog}$ ) are presented as mean (range). Riv, rivastigmine; Gal, galactamine; Don, donepezil.

(range 59-87 years) at baseline. Twenty-two were on treatment with donepezil, 22 with galantamine, and 7 with rivastigmine. The MMSE scores at the first visit were $24.2 \pm 3.6$ and at the second visit $22.9 \pm 4.2$. For demographic data, see Table 1.

CSF samples were collected by lumbar puncture. The first $12 \mathrm{~mL}$ of CSF was collected in polypropylene tube and centrifuged at $2000 \times g$ at $+4^{\circ} \mathrm{C}$ for $10 \mathrm{~min}$. The supernatant was pipette off, gently mixed to avoid possible gradient effects, and aliquoted in $2 \mathrm{~mL}$ portions in polypropylene tubes that were stored at $-80^{\circ} \mathrm{C}$ pending analyses. All CSF analyses were performed at one occasion, avoiding freezing/thawing cycles of the samples, with baseline and endpoint samples analyzed side by side on the same assay-plate. Plasma samples (EDTA) was collected, aliqouted, and stored at $-80^{\circ} \mathrm{C}$ pending analyses. As for CSF samples, baseline and endpoint samples were analyzed side by side on the same assay-plate. All patients gave informed consent to participate in the study, which was conducted according to the provisions of the Helsinki Declaration and was approved by the ethics committee at the university of Lund and Uppsala and at the Karolinska Institute, Stockholm, Sweden.

\section{CSF and plasma analyses}

The levels of $\mathrm{sA} \beta \mathrm{PP} \alpha$ and $\mathrm{sA} \beta \mathrm{PP} \beta$ in CSF were determined using MesoScaleDiscovery duplex sA $\beta P P \alpha /$ AA $\beta P P \beta$ (MSD cat \# K15120E-2). Plasma levels of $A \beta_{1-42}$ were assayed using the Innotest sandwich ELISA (Innogenetics cat \# 80177) [21]. The levels of $A \beta_{1-40}$ in CSF and plasma were determined using a sandwich ELISA (Invitrogen, cat \# KHB3482). $\mathrm{CSF}$ levels of $\mathrm{pNfH} / \mathrm{M}$ were assayed using an internally developed assay described briefly. All steps were followed by a washing step with TBS $0.1 \%$ BSA and $0.05 \%$ Tween. Monoclonal antibody SMI31, reacting with neurofilament heavy and medium with low (hence $\mathrm{pNfH} / \mathrm{M})$ and high degree of phosphorylation $[13,22]$ 

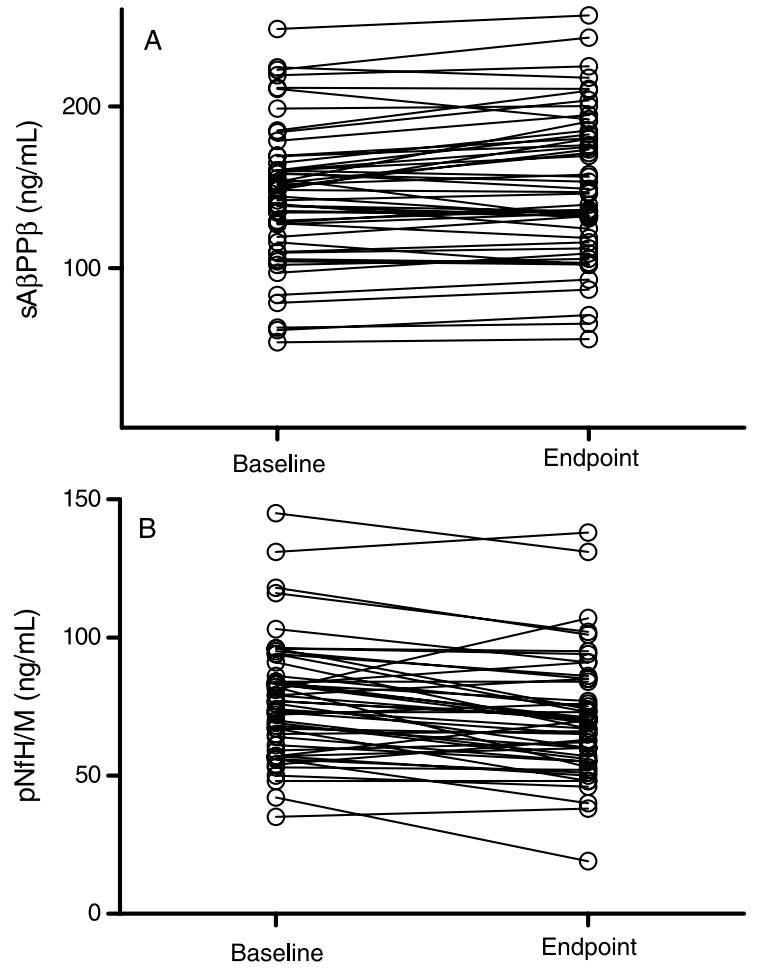

Fig. 1. Individual changes of the CSF levels of $\operatorname{sA} \beta P P \beta(A)$ and $\mathrm{pNfH} / \mathrm{M}(\mathrm{B})$ over time. A) Individual change of $\mathrm{s} A \beta P P \beta$ over time. B) Individual change of $\mathrm{pNfH} / \mathrm{M}$ over time in $\mathrm{CSF}$. Circles represent individual levels at baseline and endpoint and the change over time is illustrated by the line between the circles.

was coated in carbonate buffer $(1: 1000)$ overnight at $4^{\circ} \mathrm{C}$. The plate was blocked with $1 \%$ BSA in TBS followed by incubation of $100 \mu \mathrm{L}$ of samples for $3 \mathrm{~h}$ at room temperature (RT) while shaking. Detection antibody NA211, diluted in TBS $(1: 1000)$ was added and incubated $1 \mathrm{~h}$ at RT. A biotin labeled anti-rabbit antibody was diluted in TBS $1 \%$ BSA and incubated for $1 \mathrm{~h}$. Extravidin diluted in TBS $1 \%$ BSA was incubated for $1 \mathrm{~h}$ at RT and a subsequent amplification step using ELAST ELISA Amplification System (Perkin Elmer, cat \# NEP116001EA) was performed. After incubation with streptavidin-HRP diluted in PBS T $1 \%$ BSA and a final incubation with TMB substrate for 20 min, the reaction was quenched with $2 \mathrm{M}$ sulphuric acid and the plate was read at $570 \mathrm{~nm}$. Plasma levels of $\mathrm{sA} \beta P P \beta$ were assayed using an ELISA kit from IBL (Human sA $\beta P P \beta-w$ highly sensitive assay kit cat no 27732 IBL), according to instructions but with calibration standard range of $0.19-12.5 \mathrm{ng} / \mathrm{ml}$. The levels of $A \beta_{1-42}$ was measured in CSF from the very same patient population and presented in a previous publication [23]. All immunoassays were validated according to internal criteria.

\section{Statistics}

All concentration data were logarithmically transformed before being statistically analyzed. As a result, the estimates and confidence limits for the effects are given as percentage of the Baseline values. The within-patient Coefficients of Variation (CVs), and their corresponding confidence limits, were calculated using the within-patient variance, which in turn was obtained as the variance component due to patient, using a repeated measures linear mixed model [41]. Patient was entered as the random component of the mixed model. Gender, APOE, and timepoint (baseline and endpoint) were entered as fixed effect covariates.

The Pearson correlation coefficient was used to assess the strength of linear relationships between the different biomarkers, cognitive status at baseline, and change in CSF levels of sA $\beta P P \beta$ and $\mathrm{pNfH} / \mathrm{M}$, as well as the relationship between baseline and endpoint for the same biomarker. Scatter-plots showing the correlations are given in the results section.

All the statistical analyses for this work were generated using SAS/STAT software, Version 9.2 of the SAS System for Windows, SAS Institute Inc., Cary, NC, USA.

\section{RESULTS}

CSF

There was a $3 \%$ increase $(95 \%$ Confidence Interval $(\mathrm{CI})[1 \%, 6 \%], p=0.0193)$ in the levels of CSF sA $\beta P P \beta$ comparing baseline with endpoint (Fig. 1A) while no change was seen in levels of $\operatorname{sA} \beta P P \alpha$ $(p=0.3110)$ or $\mathrm{A} \beta_{1-40}(p=0.7483)$. There was a $9 \%$ decrease in $\mathrm{CSF}$ pNfH/M comparing baseline with endpoint (95\% CI [-15\%,-3\%], $p=0.0079$ ) (Fig. 1B). The CSF levels of $A \beta_{1-40}$ were very stable over time (Table 2).

There was a weak, but still statistically significant correlation between $A \beta_{1-40}$ and $A \beta_{1-42}$ in CSF $(p=0.0285, r=0.30695)$ at baseline and endpoint $(p=0.0162, r=0.33524)$ (data not shown). There was also a weak, but statistically significant correlation between $\mathrm{sA} \beta \mathrm{PP} \beta$ and $\mathrm{A} \beta_{1-40}(p=0.0035$, $r=0.40178)$ at baseline but no correlation at endpoint $(p=0.0511, r=0.27471)$ (data not shown). No correlation between $\mathrm{sA} \beta \mathrm{PP} \beta$ and $A \beta_{1-42}$ in CSF was 
Table 2

Biomarker levels in CSF and plasma

\begin{tabular}{|c|c|c|c|}
\hline & Baseline & Endpoint & Within-patient CV (\%) \\
\hline $\mathrm{CSF} \mathrm{sA} \beta \mathrm{PP} \alpha(\mathrm{ng} / \mathrm{mL})$ & $181.53(58.91-293.12)$ & $182.45(61.10-284.30)$ & 5.94 \\
\hline CSF sA $\beta P P \beta(n g / m L)$ & $145.64(54.00-247.89)$ & $150.71(56.12-256.38)^{\#}$ & 5.84 \\
\hline $\mathrm{CSF} A \beta_{1-40}(\mathrm{pg} / \mathrm{mL})$ & 6817 (1921-19588) & 6808 (1157-20997) & 10.14 \\
\hline $\mathrm{CSF}$ pNfH/M $(\mathrm{pg} / \mathrm{mL})^{*}$ & $77.18(35-145)$ & $70.66(19-138)^{\#}$ & 17.28 \\
\hline Plasma $A \beta_{1-40}(\mathrm{pg} / \mathrm{mL})$ & $113.97(53.90-187.48)$ & $118.09(51.9-251.85)$ & 26.37 \\
\hline Plasma $A \beta_{1-42}(\mathrm{pg} / \mathrm{mL})$ & $54.81(7.80-444.84)$ & $55.74(7.80-507.81)$ & 14.09 \\
\hline Plasma sA $\beta P P \beta(\mathrm{ng} / \mathrm{mL})^{* *}$ & $3.41(1.06-11.86)$ & $3.54(1.07-11.12)$ & 42.35 \\
\hline
\end{tabular}

Data presented as mean (range), $n=51{ }^{*} n=50,{ }^{* *} n=25$. ${ }^{*}$ indicates a statistically significant change comparing endpoint with baseline. Levels of $\mathrm{sA} \beta P P \beta$ increased by $3 \%(p=0.0193)$ and CSF levels of $\mathrm{pNfH} / \mathrm{M}$ decreased with $9 \%(p=0.0079)$.

observed at baseline $(p=0.2257, r=0.17265)$ or endpoint ( $p=0.2229, r=0.17367$ ) (data not shown). There was no correlation between $\mathrm{pNfH} / \mathrm{M}$ and $\mathrm{A} \beta_{1-42}$ in CSF at baseline $(p=0.07046, r=0.6268)$ or endpoint $(p=0.08943, r=0.5368)$ (data not shown).

Correlation tests between baseline values for MMSE and ADAS-Cog and changes in $\mathrm{SA} \beta P P \beta$ and $\mathrm{pNfH} / \mathrm{M}$ in CSF gave non-significant results (data not shown). Stratifying for the covariates $A P O E$ and gender did not affect the outcome of the correlation test performed in CSF. The mean levels and within patient CV for the biochemical biomarkers in CSF are presented in Table 2.

\section{Plasma}

There was no change between baseline and endpoint in plasma levels of $A \beta_{1-40}(p=0.7643)$ or $A \beta_{1-42}$ $(p=0.9342)$ (Table 2). Neither was there a significant change between baseline and endpoint in $\mathrm{SA} \beta \mathrm{PP} \beta$ in plasma ( $p=0.5998)$ (Table 2).

There was a weak but positive correlation between sA $\beta P P \beta$ and $\mathrm{A} \beta_{1-42}$ in plasma at baseline $(p=0.0034$, $r=0.56233)$ and endpoint $(p=0.0019, r=0.59005$ while there was no correlation between sA $\beta P P \beta$ and $\mathrm{A} \beta_{1-40}$ at baseline $(p=0.2158, r=0.25651)$ or endpoint ( $p=0.9262, r=0.01953)$ (data not shown). No correlation between $A \beta_{1-40}$ and $A \beta_{1-42}$ at baseline $(r=0.07295, p=0.6109)$ or endpoint $(p=0.5916$, $r=0.07693$ ) was observed (data not shown).

Finally, no correlation between CSF levels of $A \beta_{1-40}$ with plasma levels of $A \beta_{1-40}$ at baseline $(p=0.9170$, $r=0.01496)$ or between CSF levels of $\mathrm{A} \beta_{1-42}$ in CSF and $\mathrm{A} \beta_{1-42}$ in plasma at endpoint $(p=0.15958$, $r=0.2633$ ) was observed. As for the CSF correlations, gender and APOE4 status did not affect the outcome of any correlation. The mean levels and within patient $\mathrm{CV}$ for the biochemical biomarkers in plasma are presented in Table 2.

\section{DISCUSSION}

In the present study, the longitudinal stability of potential efficacy biomarkers was evaluated. The CSF levels of $A \beta_{1-40}, s A \beta P P \beta$, and $\mathrm{s} \beta \beta P P \alpha$ are believed to reflect $A \beta P P$ metabolism in the brain. For drugs targeting the amyloid disease pathway, these biomarkers are potential markers to demonstrate central (CSF) and peripheral (plasma) target engagement. For disease modifying drugs, biomarkers downstream of target and linked to disease pathology are potential markers to support disease modification. In the present study we have also evaluated CSF levels of $\mathrm{pNfH} / \mathrm{M}$, a marker for axonal degeneration.

We report very stable levels of $A \beta_{1-40}$ in CSF and of $A \beta_{1-40}$ and $A \beta_{1-42}$ in plasma over a 6-month period. The within patient CVs were $10.14 \%$ (Table 2), $26.37 \%$, and $14.09 \%$ (Table 2) respectively. Our data are in agreement with previous studies reporting on stable levels of $A \beta_{1-40}$ and $A \beta_{1-42}$ in plasma over 9 months [24] and 12 months [25]. There are broad interindividual variations which are also seen in previous studies [24]. These stable levels over time indicate that the biomarkers are fit to be included in early clinical trials to demonstrate peripheral target engagement. The broad inter-individual variation, however, implies that large cohorts of patients need to be included. We also report stable levels of $\mathrm{sA} \beta \mathrm{PP} \beta$ in plasma over time, with a mean baseline value of $3.41 \mathrm{ng} / \mathrm{mL}$ compared to mean levels of $3.54 \mathrm{ng} / \mathrm{mL}$ at endpoint, but with large intra-individual CV of $42.35 \%$. Part of the imprecision could be due to variations caused by the processing of blood to plasma and partly due to the complexity of the matrix, for example, less homogeneous compared to CSF.

The longitudinal stability of $A \beta_{1-40}$ in CSF is less studied compared to the stability of $A \beta_{1-42} \mathrm{CSF}$ levels and with contradictory results. Our data are supported by a previous study reporting stable CSF levels of 
$A \beta_{1-40}$ over an average time of 18.6 months [26] while in conflict with a 3-year follow up study by Tapiola and co-workers [27] which present a significant decrease of $A \beta_{1-40}$ over time. There are some possible explanations for these contradictory results. One factor could be in what stage of the disease the patients are in. The general conclusion from the many studies performed on longitudinal stability of $A \beta_{1-42}$ in CSF indicates that levels of $A \beta_{1-42}$ start to decrease very early in disease progression, before clinical symptoms [28]. The patients included in the present study have mild to moderate $\mathrm{AD}$ with a mean MMSE of 24.2. In the study by Kanai et al. [26], patients with AD had a mean MMSE of 16.7 at baseline indicating that they are in a later stage of the disease, which could partly explain the stable levels of both $A \beta_{1-40}$ and $A \beta_{1-42}$ in CSF. In the Tapiola study [27], patients had a mean MMSE of 21, indicating mild-moderate AD and in an earlier stage of the disease compared to the Kanai study. Another possible factor that could explain the contradictory outcomes is how and for how long CSF samples have been stored. The long term stability of $\mathrm{A} \beta_{1-40}$ and $\mathrm{A} \beta_{1-42}$ when storing $\mathrm{CSF}$ at $-80^{\circ} \mathrm{C}$ has been evaluated indicating stable levels of $A \beta_{1-42}$ but a small decrease in $A \beta_{1-40}$. These data suggests that $A \beta_{1-40}$ might be more prone to degradation over a sixyear period [29]. In the present study there is only a 6 month time difference between sampling, hence this should not be a problem compared to the longer studies, which is also supported by our data indicating stable levels of CSF $A \beta_{1-40}$. Finally, conflicting data could be explained by what species of $A \beta$ the ELISA is measuring, $A \beta_{1-40}$ or $A \beta$ peptides ending at amino acid (aa) $40, A \beta_{x-40}$. In the work by Tapiola [27], the 6E10 antibody was used as the N-terminal capture antibody. $6 \mathrm{E} 10$ has been demonstrated to bind between aa 4-9, indicating that in addition to $A \beta_{1-40}$, $\mathrm{N}$-terminally truncated species could be measured. In the Kanai et al. [26] study, Ban-50 antibody was used, specific for aa 1-16. Data using IP-MALDI technique does not demonstrated the presence of N-terminally truncated of $A \beta$ in CSF [7], suggesting that if there are $\mathrm{N}$-terminal truncated $\mathrm{A} \beta$, they are most probably in very low concentrations.

The CSF levels of $\mathrm{sA} \beta \mathrm{PP} \alpha$ were found to be very stable over time with a within-patient $\mathrm{CV}$ of $5.94 \%$ (Table 2). However, after this 6 month longitudinal follow-up we saw a small, but consistent and statistically significant increase in CSF levels of sA $\beta P P \beta$ (Fig. 1A). This increase could reflect a disturbed processing of $A \beta P P$ in the brain of patients with AD leading to amyloid plaque pathology. To our knowledge there are no previous reports on longitudinal stability of $\mathrm{sA} \beta \mathrm{PP} \alpha$ or $\mathrm{sA} \beta \mathrm{PP} \beta$ fragments in CSF. Previous studies have reported conflicting data comparing CSF levels between patients with $\mathrm{AD}$ and controls [30-32]. Olsson and colleagues [30] report no differences comparing AD with control while Sennvik et al. [30] report decreased levels of $\mathrm{sA} \beta \mathrm{PP} \alpha$ in patients with $\mathrm{AD}$. The third study by Lewzcuk et al. [32] demonstrated increased levels of both $\mathrm{sA} \beta P P \beta$ and $\mathrm{sA} \beta \mathrm{PP} \alpha$ in patients clinically diagnosed with $\mathrm{AD}$ and with supportive pathological CSF analyses (tauphospho tau and $A \beta_{1-42}$ ) compared to patients with $\mathrm{AD}$ and non-supportive CSF analyses. A mutually exclusive regulation of the A $\beta P P$ cleavage pathways is suggested to occur and enhanced $\alpha$-cleavage may suppress $\beta$-cleavage at the same time [33]. However, this generally accepted hypothesis does not seem to be valid under all circumstances $[34,35]$ and is supported by the clinical data in the Sennvik study [30] and the Olsson et al. study [31]. The processing of A $\beta P P$ is probably much more complicated and there is very little information of the turnover rate in brain and CSF. Further clinical studies are needed to confirm our data. Evaluating stability of a longer time period would be beneficial as well as performing pre-clinical and clinical studies with compounds targeting the A $\beta \mathrm{PP}$ processing to enable more knowledge. Since the change over time of $\mathrm{sA} \beta P P \beta$ is very small, we believe that $\mathrm{sA} \beta \mathrm{PP}$ is suitable as a biomarker to monitor treatment effects.

Since $A \beta_{1-40}$ and $A \beta_{1-42}$ are subsequent products to sA $\beta P P$ fragments during the processing of $A \beta P P$ and hence mechanistic markers of the same protein processing, we performed statistical analyses evaluating if there were any correlations between $\mathrm{sA} \beta \mathrm{PP} \beta$ (where we observe a change over time) and $A \beta_{1-40}$ and $A \beta_{1-42}$ in plasma and CSF. There was a weak but statistically significant positive correlation between $\mathrm{SA} \beta P P \beta$ and $\mathrm{A} \beta_{1-40}$ at baseline in CSF. There are no individuals with extreme values indicating outliers. There is no effect from the covariats of $A P O E$ and gender (data not shown) that could explain this positive correlation. There was also a weak but statistically significant positive correlation between $\mathrm{A} \beta \mathrm{PP} \beta$ and $A \beta_{1-42}$ found in plasma at baseline and endpoint. This weak, but statistically significant, correlation most likely is dependent on extreme values for a small number of individuals. We have carefully gone through raw data, and the individuals with high levels of $\mathrm{SA} \beta P P \beta$ also have high levels of $A \beta_{1-40}$, hence, we do not consider these individuals as outliers. There were no other demographic data indicating that these patients should be considered as outliers. However, taken together, these correlation 
data implies that there is a wide inter-individual variability and our data needs confirmation in a larger cohort of patients. As reported earlier we found a positive correlation between $A \beta_{1-40}$ and $A \beta_{1-42}$ in CSF at endpoint and baseline, indicating a similar turnover rate in this cohort of patients and in this stage of the disease.

We also evaluated the longitudinal stability of the $\mathrm{pNfH} / \mathrm{M}$ in CSF and identified a statistically significant increase of $9 \%$ comparing end point with baseline (Fig. 1B). As Nfs are confined to the nervous system, they might be one of the best markers reflecting neuronal pathogenic changes seen in some neurological disorders, such as AD. However, only a limited number of studies have reported data on Nf levels in CSF in $\mathrm{AD}[15,16,36-38]$. Only one study report on the CSF levels of pNfH/M [15] and to our knowledge this is the first time the longitudinal stability of $\mathrm{pNfH} / \mathrm{M}$ is reported. This study demonstrates that CSF levels of Nf might be a marker for brain aging as well as a neurodegenerative marker in general [15]. The followup time probably needs to be increased, compared to the six months in our study, to be able to capture any aging effects. Hu et al. [15] also demonstrate that the CSF levels of $\mathrm{pNfH} / \mathrm{M}$ are increased in $\mathrm{AD}$ compared to aged controls and patients with vascular dementia, hence $\mathrm{pNfH} / \mathrm{M}$ CSF levels are able to discriminate patients with $\mathrm{AD}$ from normal aging and other neurological conditions such as vascular dementia. This study, together with our data, suggests that measuring $\mathrm{pNfH} / \mathrm{M}$ in $\mathrm{CSF}$ is a marker for neurodegeneration and could be used as a biomarker in clinical trials to support disease modification. A previous publication has reported on the CSF levels of t-tau and p-tau, suggested markers for neurodegeneration, in this particular cohort of patients revealing very stable levels over time [23]. Both t-tau and p-tau are established diagnostic biomarkers of $\mathrm{AD}$ and they are increased 2-3-fold in CSF from patients with AD. Even though less studied, there is no such clear increase for the different forms of $\mathrm{Nfs}$ in $\mathrm{AD}$. Hence, further characterization of the Nf protein(s) in CSF together with optimized quantitative assays is needed followed by the application in clinical studies to further increase our understanding of $\mathrm{Nf}$ as a biomarker in neurodegeneration.

Since we identified a change in CSF levels of $\mathrm{pNfH}$ and $\mathrm{sA} \beta \mathrm{PP} \beta$ over time, we investigated if there was a correlation between the cognitive status at baseline (MMSE and ADAS-Cog) and the change over time in CSF. We hypothesized that the cognitive status of the patient at baseline was linked to an altered A $\beta P P$ metabolism, measured by the $\mathrm{sA} \beta P P \beta$ fragment in
CSF or a more rapid neurodegeneration, measured by the change in $\mathrm{CSF}$ pNfH/M. However, we did not see any correlation. Stratification for gender or APOE4 status did not affect the results. We also considered correlating the change over time in cognitive status to the change of $\mathrm{pNfH} / \mathrm{M}$ and $\mathrm{sA} \beta \mathrm{PP} \beta$ in CSF. However, we concluded that a 6-month follow up time is a rather short time and excluded that analysis. Previous reports indicate quite small changes in MMSE and ADAS-Cog over 1 year period. The mean decline in MMSE score has been reported to be 3-4 point over 1 year [39]. The average increase (representing cognitive deterioration) in the ADAS-Cog score has been observed at a rate of approximately 5 points per year for placebo treated patients with AD and 8-12 points for untreated patients with $\mathrm{AD}$ [40]. In the present study, the mean decrease in MMSE was 1.4 and the mean increase in ADAS-Cog was 0.4 points.

To conclude, this study reports on the longitudinal stability of biomarkers in CSF and plasma in AD. One limitation with the study is the length of the study being only 6 months, compared to the long clinical course of $\mathrm{AD}$ as well as the length of the ongoing and planned clinical Phase II and III trials. The second limitation is the fact that all patients were on stable treatment with AChE inhibitors, which could somehow affect the stability of the biomarkers evaluated. However, it is also very likely today that patients enrolled into clinical trials will be on treatment with AChE inhibitors, hence data in the present study is of relevance. The main finding was a statistically significant decrease in the CSF levels of $\mathrm{pNfH} / \mathrm{M}$ comparing endpoint with baseline. These data together with previous studies [15] indicates that measurement of $\mathrm{pNfH} / \mathrm{M}$ in $\mathrm{CSF}$ might reflect an ongoing neurodegenerative process and the possible use as a biomarker supporting disease modification claim in clinical trials. The study also reveals very stable levels of $\mathrm{sA} \beta P P \alpha$ and $A \beta_{1-40}$ in CSF and sA $\beta P P \beta, A \beta_{1-42}$, and $A \beta_{1-40}$ in plasma, suggesting the suitability as biomarkers in clinical trials. Finally the finding indicating an increase in $\triangle A \beta P P \beta$ in CSF over a 6-month time period is novel, indicating its use as a mechanistic biochemical marker to demonstrate central target engagement. Previous clinical studies on the sA $\beta P P$ fragments present conflicting data and there is a need to further evaluate the levels and utility of sA $\beta P P$ fragments in larger clinical studies.

\section{DISCLOSURE STATEMENT}

Authors' disclosures available online (http://www.jalz.com/disclosures/view.php?id=1426). 


\section{REFERENCES}

[1] Blennow K (2005) CSF biomarkers for Alzheimer's disease: Use in early diagnosis and evaluation of drug treatment. Expert Rev Mol Diagn 5, 661-672.

[2] Sjogren M, Davidsson P, Tullberg M, Minthon L, Wallin A, Wikkelso C, Granerus AK, Vanderstichele H, Vanmechelen E, Blennow K (2001) Both total and phosphorylated tau are increased in Alzheimer's disease. J Neurol Neurosurg Psychiatry 70, 624-630.

[3] Hesse C, Rosengren L, Vanmechelen E, Vanderstichele H, Jensen C, Davidsson P, Blennow K (2000) Cerebrospinal fluid markers for Alzheimer's disease evaluated after acute ischemic stroke. J Alzheimers Dis 2, 199-206.

[4] Blennow K (2005) CSF biomarkers for Alzheimer's disease: Use in early diagnosis and evaluation of drug treatment. Expert Rev Mol Diagn 5, 661-672.

[5] Fagan AM, Mintun MA, Mach RH, Lee SY, Dence CS, Shah AR, LaRossa GN, Spinner ML, Klunk WE, Mathis CA, DeKosky ST, Morris JC, Holtzman DM (2006) Inverse relation between in vivo amyloid imaging load and cerebrospinal fluid Abeta42 in humans. Ann Neurol 59, 512-519.

[6] Strozyk D, Blennow K, White LR, Launer LJ (2003) CSF Abeta 42 levels correlate with amyloid-neuropathology in a population-based autopsy study. Neurology 60, 652-656.

[7] Portelius E, Westman-Brinkmalm A, Zetterberg H, Blennow K (2006) Determination of beta-amyloid peptide signatures in cerebrospinal fluid using immunoprecipitation-mass spectrometry. J Proteome Res 5, 1010-1016.

[8] Bibl M, Welge V, Esselmann H, Wiltfang J (2012) Stability of amyloid-beta peptides in plasma and serum. Electrophoresis 33, 445-450.

[9] Du P, Wood KM, Rosner MH, Cunningham D, Tate B, Geoghegan KF (2007) Dominance of amyloid precursor protein sequence over host cell secretases in determining betaamyloid profiles studies of interspecies variation and drug action by internally standardized immunoprecipitation/mass spectrometry. J Pharmacol Exp Ther 320, 1144-1152.

[10] Segal MB (2000) The choroid plexuses and the barriers between the blood and the cerebrospinal fluid. Cell Mol Neurobiol 20, 183-196.

[11] Gresle MM, Shaw G, Jarrot B, Alexandrou EN, Friedhuber A, Kilpatrick TJ, Butzkueven H (2008) Validation of a novel biomarker for acute axonal injury in experimental autoimmune encephalomyelitis. J Neurosci Res 86, 3548-3555.

[12] Petzold A, Keir G, Green AJ, Giovannoni G, Thompson EJ (2003) A specific ELISA for measuring neurofilament heavy chain phosphoforms. J Immunol Methods 278, 179-190.

[13] Goldstein ME, Sternberger NH, Sternberger LA (1987) Phosphorylation protects neurofilaments against proteolysis. $J$ Neuroimmunol 14, 149-160.

[14] Brettschneider J, Petzold A, Schottle D, Claus A, Riepe M, Tumani H (2006) The neurofilament heavy chain (NfH) in the cerebrospinal fluid diagnosis of Alzheimer's disease. Dement Geriatr Cogn Disord 21, 291-295.

[15] Hu YY, He SS, Wang XC, Duan QH, Khatoon S, Iqbal K, Grundke-Iqbal I, Wang JZ (2002) Elevated levels of phosphorylated neurofilament proteins in cerebrospinal fluid of Alzheimer disease patients. Neurosci Lett 320, 156-160.

[16] Rosengren LE, Karlsson JE, Sjogren M, Blennow K, Wallin A (1999) Neurofilament protein levels in CSF are increased in dementia. Neurology 52, 1090-1093.

[17] America Psychiatric Association (1994) Diagnostic and Statistical Manual of Mental Disorders, 4th ed. Washington, DC.
[18] McKhann G, Drachman D, Folstein M, Katzman R, Price D, Stadlan EM (1984) Clinical diagnosis of Alzheimer's disease: Report of the NINCDS-ADRDA Work Group under the auspices of Department of Health and Human Services Task Force on Alzheimer's Disease. Neurology 34, 939-944.

[19] Folstein MF, Folstein SE, McHugh PR (1975) "Mini-mental state". A practical method for grading the cognitive state of patients for the clinician. J Psychiatr Res 12, 189-198.

[20] Mohs RC, Cohen L (1988) Alzheimer's Disease Assessment Scale (ADAS). Psychopharmacol Bull 24, 627-628.

[21] Vanderstichele H, Van Kerschaver E, Hesse C, Davidsson P, Buyse MA, Andreasen N, Minthon L, Wallin A, Blennow K, Vanmechelen E (2000) Standardization of measurement of beta-amyloid(1-42) in cerebrospinal fluid and plasma. Amyloid 7, 245-258.

[22] Goldstein ME, Sternberger LA, Sternberger NH (1987) Varying degrees of phosphorylation determine microheterogeneity of the heavy neurofilament polypeptide (Nf-H). J Neuroimmunol 14, 135-148.

[23] Blennow K, Zetterberg H, Minthon L, Lannfelt L, Strid S, Annas P, Basun H, Andreasen N (2007) Longitudinal stability of CSF biomarkers in Alzheimer's disease. Neurosci Lett 419 , 18-22.

[24] Hoglund K, Wiklund O, Vanderstichele H, Eikenberg O, Vanmechelen E, Blennow K (2004) Plasma levels of betaamyloid(1-40), beta-amyloid(1-42), and total beta-amyloid remain unaffected in adult patients with hypercholesterolemia after treatment with statins. Arch Neurol 61, 333-337.

[25] Hoglund K, Thelen KM, Syversen S, Sjogren M, von Bergmann K, Wallin A, Vanmechelen E, Vanderstichele H, Lutjohann D, Blennow K (2005) The effect of simvastatin treatment on the amyloid precursor protein and brain cholesterol metabolism in patients with Alzheimer's disease. Dement Geriatr Cogn Disord 19, 256-265.

[26] Kanai M, Matsubara E, Isoe K, Urakami K, Nakashima K, Arai H, Sasaki H, Abe K, Iwatsubo T, Kosaka T, Watanabe M, Tomidokoro Y, Shizuka M, Mizushima K, Nakamura T, Igeta Y, Ikeda Y, Amari M, Kawarabayashi T, Ishiguro K, Harigaya Y, Wakabayashi K, Okamoto K, Hirai S, Shoji M (1998) Longitudinal study of cerebrospinal fluid levels of tau, A beta1-40, and A beta1-42(43) in Alzheimer's disease: A study in Japan. Ann Neurol 44, 17-26.

[27] Tapiola T, Pirttila T, Mikkonen M, Mehta PD, Alafuzoff I, Koivisto K, Soininen H (2000) Three-year follow-up of cerebrospinal fluid tau, beta-amyloid 42 and 40 concentrations in Alzheimer's disease. Neurosci Lett 280, 119-122.

[28] Blennow K, Hampel H, Weiner M, Zetterberg H (2010) Cerebrospinal fluid and plasma biomarkers in Alzheimer disease. Nat Rev Neurol 6, 131-144.

[29] Schipke CG, Jessen F, Teipel S, Luckhaus C, Wiltfang J, Esselmann H, Frolich L, Maier W, Ruther E, Heppner FL, Prokop S, Heuser I, Peters O (2011) Long-term stability of Alzheimer's disease biomarker proteins in cerebrospinal fluid. $J$ Alzheimers Dis 26, 255-262.

[30] Sennvik K, Fastbom J, Blomberg M, Wahlund LO, Winblad B, Benedikz E (2000) Levels of alpha- and beta-secretase cleaved amyloid precursor protein in the cerebrospinal fluid of Alzheimer's disease patients. Neurosci Lett 278, 169-172.

[31] Olsson A, Hoglund K, Sjogren M, Andreasen N, Minthon L, Lannfelt L, Buerger K, Moller HJ, Hampel H, Davidsson P, Blennow K (2003) Measurement of alpha- and beta-secretase cleaved amyloid precursor protein in cerebrospinal fluid from Alzheimer patients. Exp Neurol 183, 74-80.

[32] Lewczuk P, Kamrowski-Kruck H, Peters O, Heuser I, Jessen F, Popp J, Burger K, Hampel H, Frolich L, Wolf S, Prinz B, 
Jahn H, Luckhaus C, Perneczky R, Hull M, Schroder J, Kessler H, Pantel J, Gertz HJ, Klafki HW, Kolsch H, Reulbach U, Esselmann H, Maler JM, Bibl M, Kornhuber J, Wiltfang J (2010) Soluble amyloid precursor proteins in the cerebrospinal fluid as novel potential biomarkers of Alzheimer's disease: A multicenter study. Mol Psychiatry 15, 138-145.

[33] Checler F (1995) Processing of the beta-amyloid precursor protein and its regulation in Alzheimer's disease. J Neurochem 65, 1431-1444.

[34] Tomita S, Kirino Y, Suzuki T (1998) A basic amino acid in the cytoplasmic domain of Alzheimer's beta-amyloid precursor protein (APP) is essential for cleavage of APP at the alpha-site. J Biol Chem 273, 19304-19310.

[35] Mills J, Reiner PB (1999) Regulation of amyloid precursor protein cleavage. J Neurochem 72, 443-460.

[36] Brettschneider J, Petzold A, Sussmuth SD, Ludolph AC Tumani H (2006) Axonal damage markers in cerebrospinal fluid are increased in ALS. Neurology 66, 852-856.
[37] Pijnenburg YA, Janssen JC, Schoonenboom NS, Petzold A, Mulder C, Stigbrand T, Norgren N, Heijst H, Hack CE, Scheltens P, Teunissen CE (2007) CSF neurofilaments in frontotemporal dementia compared with early onset Alzheimer's disease and controls. Dement Geriatr Cogn Disord 23, 225230.

[38] Sjogren M, Rosengren L, Minthon L, Davidsson P, Blennow $\mathrm{K}$, Wallin A (2000) Cytoskeleton proteins in CSF distinguish frontotemporal dementia from AD. Neurology 54, 19601964.

[39] Teri L, Hughes JP, Larson EB (1990) Cognitive deterioration in Alzheimer's disease: Behavioral and health factors. $J$ Gerontol 45, P58-P63.

[40] Mohs RC, Cohen L (1988) Alzheimer's Disease Assessment Scale (ADAS). Psychopharmacol Bull 24, 627-628.

[41] Littell, RC, Stroup WW, Freund RJ (2002) SAS for Linear Models, 4th ed., SAS Institute, Inc., Cary, NC. 